\title{
Trigger finger: etiology, evaluation, and treatment
}

\author{
Al Hasan Makkouk - Matthew E. Oetgen · \\ Carrie R. Swigart · Seth D. Dodds
}

Published online: 27 November 2007

(C) Humana Press 2007

\begin{abstract}
Trigger finger is a common finger aliment, thought to be caused by inflammation and subsequent narrowing of the A1 pulley, which causes pain, clicking, catching, and loss of motion of the affected finger. Although it can occur in anyone, it is seen more frequently in the diabetic population and in women, typically in the fifth to sixth decade of life. The diagnosis is usually fairly straightforward, as most patients complain of clicking or locking of the finger, but other pathological processes such as fracture, tumor, or other traumatic soft tissue injuries must be excluded. Treatment modalities, including splinting, corticosteroid injection, or surgical release, are very effective and are tailored to the severity and duration of symptoms.
\end{abstract}

Keywords Trigger finger - Conservative treatment . Percutaneous release Surgical management

\section{Introduction}

The malady trigger finger earns its name from the painful popping or clicking sound elicited by flexion and extension of the involved digit. First described by Notta in 1850 [1], it is caused by a difference in diameters of a flexor tendon and its retinacular sheath due to thickening and narrowing of the sheath. Though often referred to as stenosing

A. H. Makkouk

Yale University School of Medicine, 333 Cedar Street,

New Haven, CT 06510, USA

M. E. Oetgen · C. R. Swigart · S. D. Dodds $(\bowtie)$

Department of Orthopaedics and Rehabilitation, Yale University

School of Medicine, 800 Howard Avenue, P.O. Box 208071,

New Haven, CT 06520-8071, USA

e-mail: seth.dodds@yale.edu tenosynovitis [2-4], histologic studies have shown that the pathologic inflammatory changes localize specifically to the tendon sheath (tendovagina) and not the tenosynovium [5]. In light of this, the term tendovaginitis has been proposed as a more appropriate description of trigger finger [6].

\section{Pathophysiology}

In trigger finger, inflammation and hypertrophy of the retinacular sheath progressively restricts the motion of the flexor tendon [7, 8]. This sheath normally forms a pulley system comprised of a series of annular and cruciform pulleys in each digit that serve to maximize the flexor tendon's force production and efficiency of motion [9]. (Fig. 1) The first annular pulley (A1) at the metacarpal head is by far the most often affected pulley in trigger finger, though cases of triggering have been reported at the second and third annular pulleys (A2 and $\mathrm{A} 3$, respectively), as well as the palmar aponeurosis [10].

Due to its location, the A1 pulley is subjected to the highest forces and pressure gradients during normal as well as power grip [10]. The repeated friction and resulting intratendious swelling caused by movement of the flexor tendon through the A1 pulley has been compared to the fraying at the ends of a string after repeated threading through the eye of a needle [11]. Microscopic examination of trigger A1 pulleys have long shown degeneration and inflammatory cell infiltrate [5], but recent ultrastructural comparisons of normal and trigger A1 pulleys may have elucidated what may be a key phase in the pathogenesis of trigger finger. Studies using scanning and transmission electron microscopes to examine the gliding surface of the A1 pulleys demonstrated that normal specimens had an 


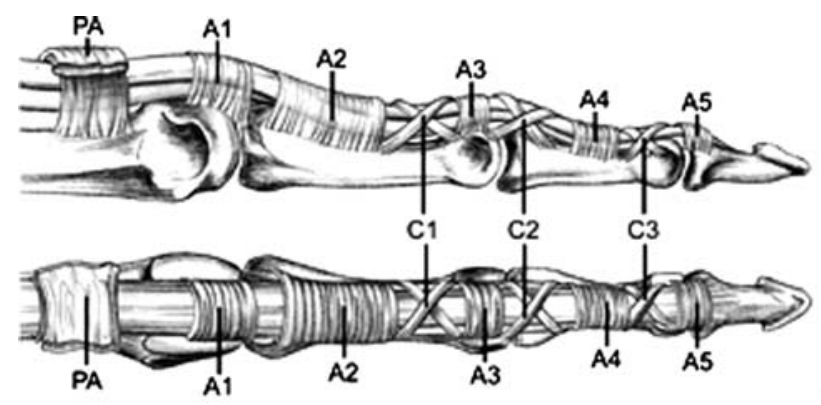

Fig. 1 Schematic of the fibro-osseus tunnel composed of five annular and three cruciform pulleys through which the flexor tendons run. The most common location of triggering is at the A1 pulley. (Adapted with permission from Berger RA, Weiss AC: Hand Surgery, Baltimore, MD, Lippincott, Williams, Wilkins, 2003)

amorphous extracellular matrix, including chondrocytes, coating the pulley's entire innermost layer. Pathologic samples had a similar general appearance, but with varying sized and shaped areas of extracellular matrix loss. These areas were characterized by chondrocyte proliferation and type III collagen production [12]. It has thus been postulated that this fibrocartilagenous metaplasia results from the repeated friction and compression between the flexor tendon and the corresponding inner layer of the A1 pulley [8].

\section{Etiology}

Several causes of trigger finger have been proposed, though the precise etiology has not been elucidated. Understandably, repetitive finger movements and local trauma are possibilities [13-15], with such stress and degenerative force also accounting for an increased incidence of trigger finger in the dominant hand $[8,16]$. There are reports linking trigger finger to occupations requiring extensive gripping and hand flexion, such as use of shears or hand held tools [5, 14, 17]. This relationship is questionable, however, with studies finding no association between trigger finger and the workplace [18, 19]. In reality the causes of trigger finger are multiple and in each individual often multifactorial.

\section{Incidence}

Primary trigger finger occurs most commonly in the middle fifth to sixth decades of life and up to 6 times more frequently in women than men $[5,7,16,20]$, although the reasons for this age and sex predilection are not entirely clear [21]. The lifetime risk of trigger finger development is between 2 and 3\%, but increases to up to $10 \%$ in diabetics $[22,23]$. The incidence in diabetics is associated with actual duration of the disease, not with glycemic control [24]. This also appears to be a higher risk for trigger finger development in patients with carpal tunnel syndrome, de Quervain's disease, hypothyroidism, rheumatoid arthritis, renal disease, and amyloidosis [24-27]. The ring finger is most commonly affected, followed by the thumb (trigger thumb), long, index, and small fingers in patients with multiple trigger digits $[21,28]$.

\section{Presentation}

The initial complaint associated with trigger finger may be of a painless clicking with digital manipulation. Further development of the condition can cause the catching or popping to become painful with both flexion and extension, and be related as occurring at either the metacarpophalangeal (MCP) or PIP joints. Other patients may notice a feeling of stiffness and then progressive loss of full flexion and/or extension of the affected digit without ever developing the catching and locking of a "typical" trigger finger. A painful nodule, a result of intratendinous swelling, may be palpated in the palmar MCP area. The patient may report MCP stiffness or swelling in the morning, or that they awaken with the digit locked and that it loosens throughout the day. A history of recent trauma to the area may also be reported. With continued deterioration the finger may present locked in flexion, requiring passive manipulation to achieve full extension. This occurs because the flexor mechanisms of the digit are generally strong enough to overcome the restrictive and narrowed retinacular sheath, while the extensors are not. Over time, the patient's desire to avoid the painful triggering caused by manipulation or use of the involved digit may lead to the development of secondary PIP contractures and digital stiffness.

\section{Diagnosis}

The classic presentation of popping and locking of a trigger finger is typically all that is needed for diagnosis; however, with acute onset of symptoms patients may present with pain and swelling over the involved flexor sheath with avoidance of finger motion. In these cases, the classic popping and triggering are not seen and the diagnosis of trigger finger must be differentiated from infection or some other traumatic injury. If desired, the diagnosis may be confirmed with an injection of lidocaine into the flexor sheath, which should relieve the pain associated with the triggering and allow the digit to become actively or passively extended. There is no role for imaging in diagnosis, with $\mathrm{x}$-rays considered unnecessary in patients without history of inflammatory disease or trauma [29]. 
The finding of a locking digit is not unique to trigger finger, and can be associated with dislocation, Dupuytren's contracture, focal dystonia, flexor tendon/sheath tumor, sesamoid bone anomalies, post-traumatic tendon entrapment on the metacarpal head, and even hysteria. The differential diagnosis of pain at the MCP joint includes de Quervain's tenosynovitis (for trigger thumb only), ulnar collateral ligament injury of the thumb (gamekeeper's thumb), MCP joint sprain, extensor apparatus injury, and MCP osteoarthritis [30-34]. Imaging with ultrasound or MRI may help with these diagnoses in atypical presentations of trigger finger.

\section{Conservative treatment}

Initial management of trigger finger is conservative and involves activity modification [35], non-steroidal antiinflammatory drugs for pain control, MCP joint immobilization, and corticosteroid injection.

\section{Splinting}

The goal of splinting is to prevent the friction caused by flexor tendon movement through the affected A1 pulley until the inflammation there resolves [10]. It is generally considered that splinting is an appropriate treatment option in patients who refuse or wish to avoid corticosteroid injection. A study of manual workers with distal interphalangeal (DIP) joint splints in full extension for 6 weeks demonstrated abatement of symptoms in over $50 \%$ of the patients [36]. In another study, splints of the MCP joint at 15 degrees of flexion (leaving the PIP and DIP joints free) were shown to provide resolution of symptoms in $65 \%$ of patients at 1-year follow-up [16]. For patients who are most bothered by symptoms of locking in the morning, splinting the PIP joint at night can be effective. Splinting yields lower success rates in patients with severe triggering or longstanding duration of symptoms.

\section{Corticosteroid injection}

Injection of corticosteroids for treatment of trigger finger was described as early as 1953 [37]. It should be attempted before surgical intervention as it is very efficacious (up to 93\%) [38], especially in non-diabetic patients with recent onset of symptoms and one affected digit with a palpable nodule [39]. It is believed that corticosteroid injection is less successful in patients with longstanding disease ( $>6$ months duration), diabetes mellitus, and multiple digit involvement as it is unable to reverse the changes of

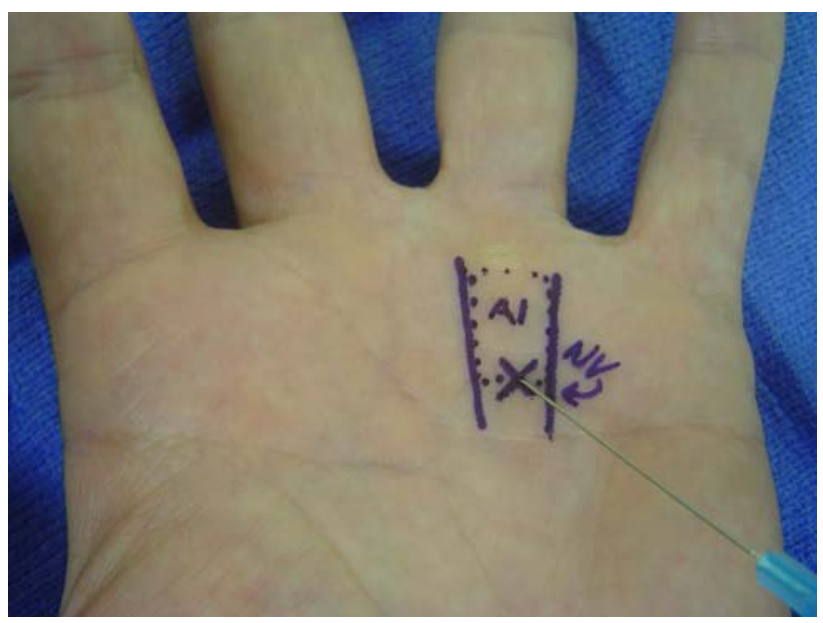

Fig. 2 Clinical photograph demonstrating the proper site for a trigger finger injection. (A1: location of the A1 pulley, NV: location of the neurovascular bundle flanking the A1 pulley)

chondroid metaplasia that take place at the A1 pulley. The injection is traditionally given directly into the sheath, however, reports of extrasynovial injection show that it may be as effective, while reducing risk of tendon damage [40, 41]. (Fig. 2) Tendon rupture is a very rare complication, with only one case being reported [42]. Other complications include dermal atrophy, fat necrosis, skin hypopigmentation, transient elevation of serum glucose in diabetic patients [43], and infection [10, 35]. If symptoms do not resolve after the first injection, or recur afterwards, a second injection is typically half as likely to succeed as the initial treatment [25].

\section{Surgical treatment}

Operative treatment, whether by percutaneous or open release, is highly successful and widely regarded as the ultimate treatment for trigger finger. Indication for surgical treatment is generally failure of conservative treatment to resolve pain and symptoms. The timing of surgery is somewhat controversial with data suggesting surgical consideration after failure of both a single as well as multiple corticosteroid injections $[44,45]$.

The percutaneous trigger finger release has been described and was first introduced by Lorthioir in 1958 [46]. In this procedure, the MCP joint is hyperextended with the palm up, thus stretching out the A1 pulley and shifting the neurovascular structures dorsally. After ethyl chloride is sprayed and lidocaine injected for pain management, a needle is inserted through the skin and onto the A1 pulley. It is then swept to slice the pulley proximal and distal to the injection site. Success rates have been reported as over $90 \%$ with this procedure [39]; however, use of this 
Fig. 3 (a) Intra-operative photo showing a thickened A1 pulley prior to release. (b) Once the A1 pulley is released the flexor tendons can be lifted out of the wound
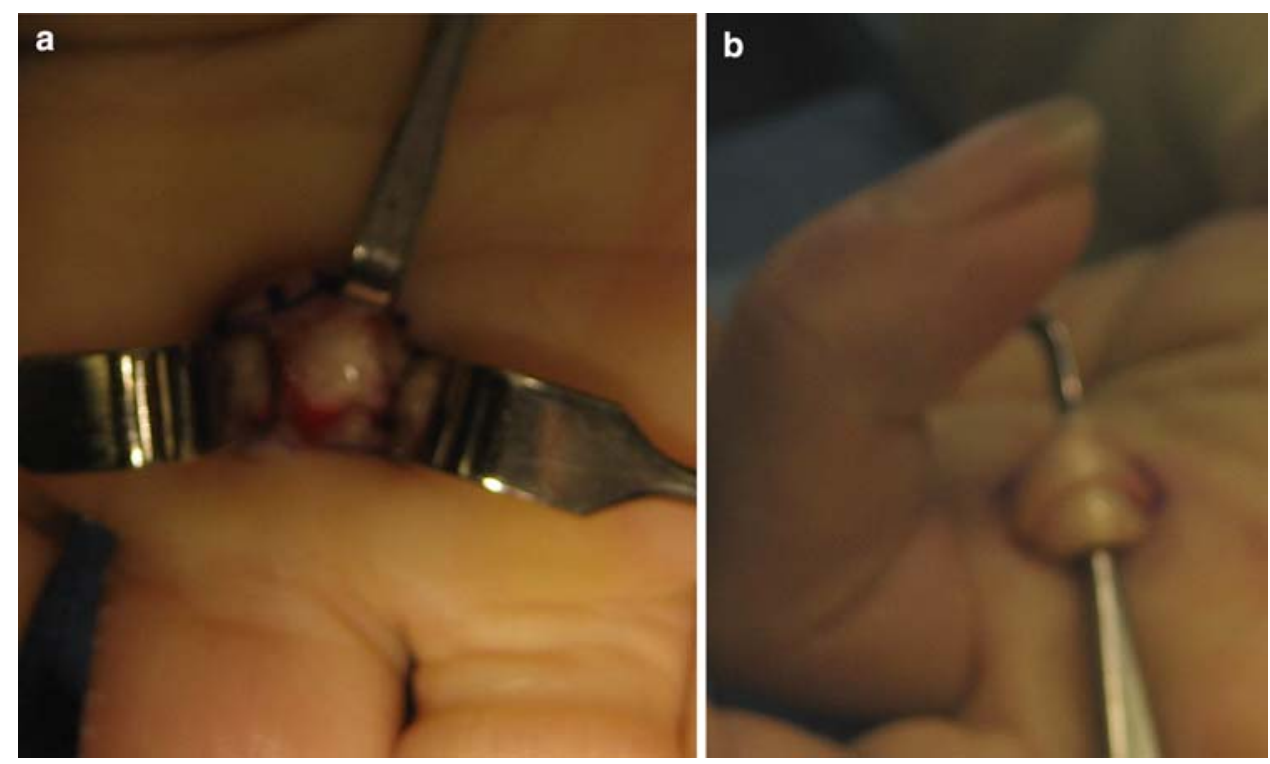

technique is tempered by the risk of digital nerve or artery injury. Other complications, including tendon bowstringing, infection, and pain are less common [47-49].

Open release of trigger finger has been used as treatment for over a century [35]. (Fig. 3 a,b) The aim of the procedure is generally the same as with the percutaneous release, which is full sectioning of the A1 pulley. The open release provides greater exposure and may be safer with regard to iatrogenic neurovascular injury. Reported success rates range from $90 \%$ to $100 \%$ proving the efficacy of this procedure. Overall complication rates may be slightly higher than with the percutaneous release, including reflex sympathetic dystrophy, infection, stiffness, nerve transection, incision pain, flexion deformity, flexor tendon bowstringing, and recurrence $(3 \%)[10,39,50]$, but in general this procedure is safe and effective.

\section{Conclusion}

Trigger finger is a long recognized condition characterized by a sometimes painful locking of the digit on flexion and extension. It is caused by the inflammation and subsequent narrowing of the A1 pulley through which the flexor tendon passes at the metacarpal head, leading to restricted movement of the tendon through the pulley. It is much more common in women than men, may be related to occupations involving constant gripping or repetitive local trauma and appears to be associated with systemic inflammatory diseases. The diagnosis is typically made by the characteristic presentation and findings on exam, and first-line treatment includes splinting and corticosteroid injections. Surgical management of this condition is indicated with recurrence after or failure of conservative management or initially in cases of $>6$ months duration and is highly effective with low complication and recurrence rates.

\section{References}

1. Notta A. Recherches sur une affection particuliere des gaines tendineuses de la main. Arch Gen Med 1850;24:142.

2. Carlson CS Jr, Curtis RM. Steroid injection for flexor tenosynovitis. J Hand Surg [Am] 1984;9:286-7.

3. Rayan GM. Distal stenosing tenosynovitis. J Hand Surg [Am] 1990;15:973-5.

4. Rhoades CE, Gelberman RH, Manjarris JF. Stenosing tenosynovitis of the fingers and thumb. Results of a prospective trial of steroid injection and splinting. Clin Orthop Relat Res 1984; 190:236-8

5. Fahey JJ, Bollinger JA. Trigger-finger in adults and children. J Bone Joint Surg Am 1954;36:1200-18.

6. Burman M. Stenosing tendovaginitis of the dorsal and volar compartments of the wrist. Arch Surg 1952;65:752-62.

7. Newport ML, Lane LB, Stuchin SA. Treatment of trigger finger by steroid injection. J Hand Surg [Am] 1990;15:748-50.

8. Sampson SP, Badalamente MA, Hurst LC et al. Pathobiology of the human A1 pulley in trigger finger. J Hand Surg [Am] 1991;16:714-21.

9. Lin GT, Amadio PC, An KN et al. Functional anatomy of the human digital flexor pulley system. J Hand Surg [Am] 1989; 14:949-56

10. Akhtar S, Bradley MJ, Quinton DN et al. Management and referral for trigger finger/thumb. BMJ 2005;331:30-3.

11. Hueston JT, Wilson WF. The aetiology of trigger finger explained on the basis of intratendinous architecture. Hand 1972;4:257-60.

12. Sbernardori MC, Mazzarello V, Tranquilli-Leali P. Scanning electron microscopic findings of the gliding surface of the A1 pulley in trigger fingers and thumbs. J Hand Surg [Br] 2007; 32:384-7.

13. Ametewee K. Trigger thumb in adults after hyperextension injury. Hand 1983;15:103-5.

14. Bonnici AV, Spencer JD. A survey of 'trigger finger' in adults. J Hand Surg [Br] 1988;13:202-3. 
15. Verdon ME. Overuse syndromes of the hand and wrist. Prim Care Clin Office Pract 1996;23:305-19.

16. Patel MR, Bassini L. Trigger fingers and thumb: when to splint, inject, or operate. J Hand Surg [Am] 1992;17:110-13.

17. Gorsche R, Wiley JP, Renger R et al. Prevalence and incidence of stenosing flexor tenosynovitis (trigger finger) in a meat-packing plant. J Occup Environ Med 1998;40:556-60.

18. Anderson B, Kaye S. Treatment of flexor tenosynovitis of the hand ('trigger finger') with corticosteroids. A prospective study of the response to local injection. Arch Intern Med 1991;151: $153-6$.

19. Trezies AJ, Lyons AR, Fielding K et al. Is occupation an aetiological factor in the development of trigger finger? J Hand Surg [Br] 1998;23:539-40.

20. Weilby A. Trigger finger. Incidence in children and adults and the possibility of a predisposition in certain age groups. Acta Orthop Scand 1970;41:419-27.

21. Bunnell S. Injuries of the hand. In: Surgery of the hand. Philadelphia: JB Lippincott; 1944.

22. Stahl S, Kanter Y, Karnielli E. Outcome of trigger finger treatment in diabetes. J Diabetes Complicat 1997;11:287-90.

23. Strom L. Trigger finger in diabetes. J Med Soc NJ 1977;74: 951-4.

24. Chammas M, Bousquet P, Renard E et al. Dupuytren's disease, carpal tunnel syndrome, trigger finger, and diabetes mellitus. J Hand Surg [Am] 1995;20:109-14.

25. Clark DD, Ricker JH, MacCollum MS. The efficacy of local steroid injection in the treatment of stenosing tenovaginitis. Plast Reconstr Surg 1973;51:179-80.

26. Griggs SM, Weiss AP, Lane LB et al. Treatment of trigger finger in patients with diabetes mellitus. J Hand Surg [Am] 1995; 20:787-9.

27. Uotani K, Kawata A, Nagao M et al. Trigger finger as an initial manifestation of familial amyloid polyneuropathy in a patient with Ile107Val TTR. Intern Med 2007;46:501-4.

28. Moore JS. Flexor tendon entrapment of the digits (trigger finger and trigger thumb). J Occup Environ Med 2000;42:526-45.

29. Katzman BM, Steinberg DR, Bozentka DJ et al. Utility of obtaining radiographs in patients with trigger finger. Am J Orthop 1999;28:703-5.

30. Kalms SB, Hojgaard AD. Trigger finger: report of an unusual case. J Trauma 1991;31:582-3.

31. Laing PW. A tendon tumour presenting as a trigger finger. J Hand Surg [Br] 1986;11:275.

32. Lapidus PW. Stenosing tenovaginitis. Surg Clin North Am 1953; 33:1317-47.
33. Oni OO. A tendon sheath tumour presenting as trigger finger. J Hand Surg [Br] 1984;9:340.

34. Seybold EA, Warhold LG. Impingement of the flexor pollicis longus tendon by an enlarged radial sesamoid causing trigger thumb: a case report. J Hand Surg [Am] 1996;21:619-20.

35. Ryzewicz M, Wolf JM. Trigger digits: principles, management, and complications. J Hand Surg [Am] 2006;31:135-46.

36. Rodgers JA, McCarthy JA, Tiedeman JJ. Functional distal interphalangeal joint splinting for trigger finger in laborers: a review and cadaver investigation. Orthopedics 1998;21:305-9, discussion 309-10.

37. Howard LD Jr, Pratt DH, Bunnell S. The use of compound F (hydrocortone) in operative and non-operative conditions of the hand. J Bone Joint Surg Am 1953;35:994-1002.

38. Freiberg A, Mulholland RS, Levine R. Nonoperative treatment of trigger fingers and thumbs. J Hand Surg [Am] 1989;14:553-8.

39. Green D, Hotchkiss R, Pederson W, et al. Tenosynovitis. In: Green's operative hand surgery. 5th ed. London: Churchill Livingstone; 2005.

40. Kazuki K, Egi T, Okada M et al. Clinical outcome of extrasynovial steroid injection for trigger finger. Hand Surg 2006; 11:1-4.

41. Taras JS, Raphael JS, Pan WT et al. Corticosteroid injections for trigger digits: is intrasheath injection necessary? J Hand Surg [Am] 1998;23:717-22.

42. Taras JS, Iiams GJ, Gibbons $M$ et al. Flexor pollicis longus rupture in a trigger thumb: a case report. J Hand Surg [Am] 1995; 20:276-7.

43. Wang AA, Hutchinson DT. The effect of corticosteroid injection for trigger finger on blood glucose level in diabetic patients. J Hand Surg [Am] 2006;31:979-81.

44. Anderson BC. Office orthopedics for primary care: diagnosis and treatment. Philadelphia: WB Saunders Company; 1999.

45. Benson LS, Ptaszek AJ. Injection versus surgery in the treatment of trigger finger. J Hand Surg [Am] 1997;22:138-44.

46. Lorthioir J. Surgical treatment of trigger finger by a subcutaneous method. J Bone Joint Surg Am 1958;40:793-5.

47. Bain GI, Turnbull J, Charles MN et al. Percutaneous A1 pulley release: a cadaveric study. J Hand Surg [Am] 1995;20:781-4, discussion 785-6.

48. Park MJ, Oh I, Ha KI. A1 pulley release of locked trigger digit by percutaneous technique. J Hand Surg [Br] 2004;29:502-5.

49. Pope DF, Wolfe SW. Safety and efficacy of percutaneous trigger finger release. J Hand Surg [Am] 1995;20:280-3.

50. Lapidus PW, Guidotti FP. Stenosing tenovaginitis of the wrist and fingers. Clin Orthop 1972;83:87-90. 